\title{
TEMPORAL VARIATIONS OF THE EARTHQUAKE DATA IN THE CATALOGUE OF SEISMICITY OF NEW ZEALAND.
}

\author{
F. R. Zúñiga ${ }^{1}$, M. Reyners ${ }^{2}$ and P. Villamor ${ }^{2}$
}

\begin{abstract}
The authors have analyzed the main temporal characteristics of the earthquake data in the catalogue of seismicity of New Zealand with the objective of providing a general overview of its content and limitations. To this end we have employed different statistical tools which allow for the objective estimate of times of changes in the seismicity rates as well as providing the main reasons for those changes. We have found that the seismicity record of the largest events $(M>4.0)$ is bracketed by significant changes which occurred during 1940, 1965-1968, and 1987 with other less significant changes taking place during 1960, 1983 and 1992.
\end{abstract}

\begin{abstract}
By comparing the rates obtained for intervals bounded by the aforementioned dates we were able to determine that a linear correction to the magnitudes in the period 1968 to 1987 for the whole depth range of events may be useful in order to match the frequency-magnitude distribution which is obtained using the current data in the interval 1987 - 2004. A different pattern emerged when separating shallow from intermediate and deep events and we found that most of the temporal variations observed affected mainly the deep events $(Z>40 \mathrm{~km})$, and that the reported rate of shallow seismicity for $M>4.0$ has been remarkably homogeneous since 1940 . This observation is supported not only by the constancy of seismicity rates but by the similarity of frequency-magnitude distributions during the time intervals analyzed. A systematic evaluation of minimum magnitude of completeness for shallow seismicity yielded values of $M_{c}=4.4$ for the interval 1940 to $1968, M_{c}=3.9$ for the interval 1968 to 1987 and $M_{c}=$ 2.6 for the current stage from 1987 to 2004 . A simple magnitude shift of 0.2 units applied to data in the interval 1968 to 1987 for intermediate and deep events was found to provide a good match to the observed rate for events from 1987 to 2004 . The evaluation of $M_{c}$ for events in the $40 \leq \mathrm{z} \leq 600 \mathrm{~km}$ range yielded values of 5.5 for data in the interval 1940 to $1968,4.0$ for 1968 to 1987 , and 3.6 for the current operative practice starting in 1987. A detailed investigation of the variation of the magnitude of completeness with time was carried out resulting in different trends which seem to correspond to the time when major changes were carried out in the network, in particular with the installation and removal of local microearthquake networks.
\end{abstract}

\section{INTRODUCTION}

The catalogue of seismicity of New Zealand (Figures 1 and 2 ) is currently updated and maintained by GeoNet, and results from the collaboration between the Institute of Geological and Nuclear Sciences Ltd., the Earthquake Commission and the Foundation for Research, Science and Technology of New Zealand.

This data set is the main source of information for all earthquake hazard investigations and mitigation projects focused on New Zealand, so its importance cannot be overemphasized. The catalogue, as it stands today, is the result of a vast number of projects and efforts through the years, as is usually the case with most seismicity catalogs, and thus it is bound to be subject to variations in its content as equipment type, network density, data processing and other resources changed and were ultimately improved to their current modern standards. As we write, equipment and technology changes day by day, so data output is subject to continuing changes.

The intention of this paper is to provide users of the catalogue with a consistent report on overall temporal characteristics and variations of earthquake data in the catalogue, as it stands at the moment of writing. The spatial characteristics of the catalogue are the subject of another article. There have been other studies dealing with some of the issues addressed here (e.g. McGinty,

\footnotetext{
'Centro de Geociencias, Universidad Nacional Autónoma de México

${ }^{2}$ Institute of Geological and Nuclear Sciences Ltd., Lower Hutt
} 
2001: Smith, 2000; Dowrick and Rhoades, 1998. Anderson and Webb, 1994) but their scope has been focused on a particular point. Ou objective, however, is to provide a generalized view of the capabilities and limitations of the content of the catalogue with hope that this can be useful even to researchers who make use of this data set on a daily basis.

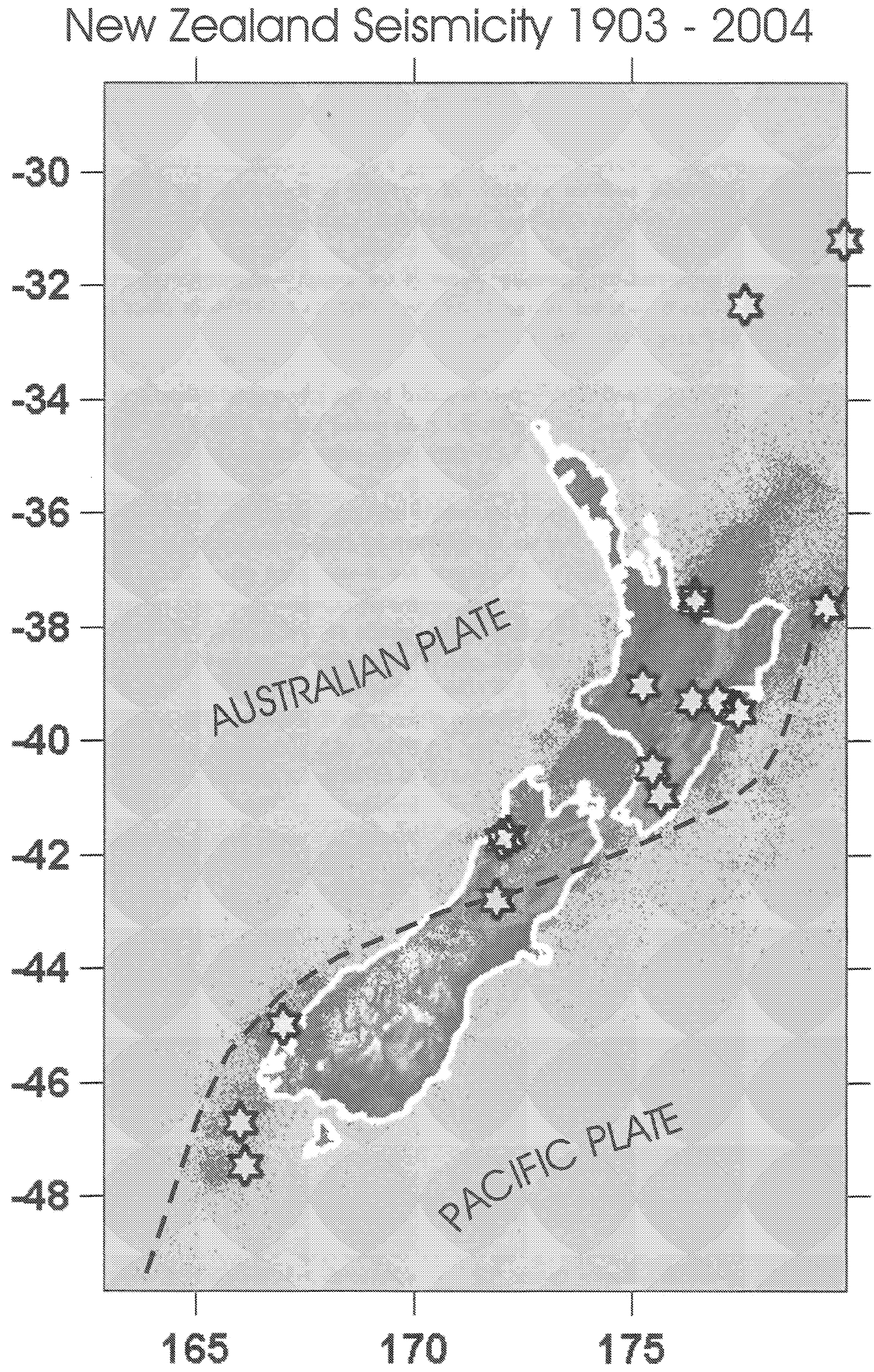

Figure 1. Epicenters (red dots) of earthquakes with $M \geq 3.0$ from the catalogue of seismicity of New Zealand. Time span is 7903 to beginning of 2004. Starts indicate events with $M \geq 7.0$. Purple lines show crustal faults. The dash line shows the main tectonic plate boundary. 
New Zealand Seismicity 2001 - 2003

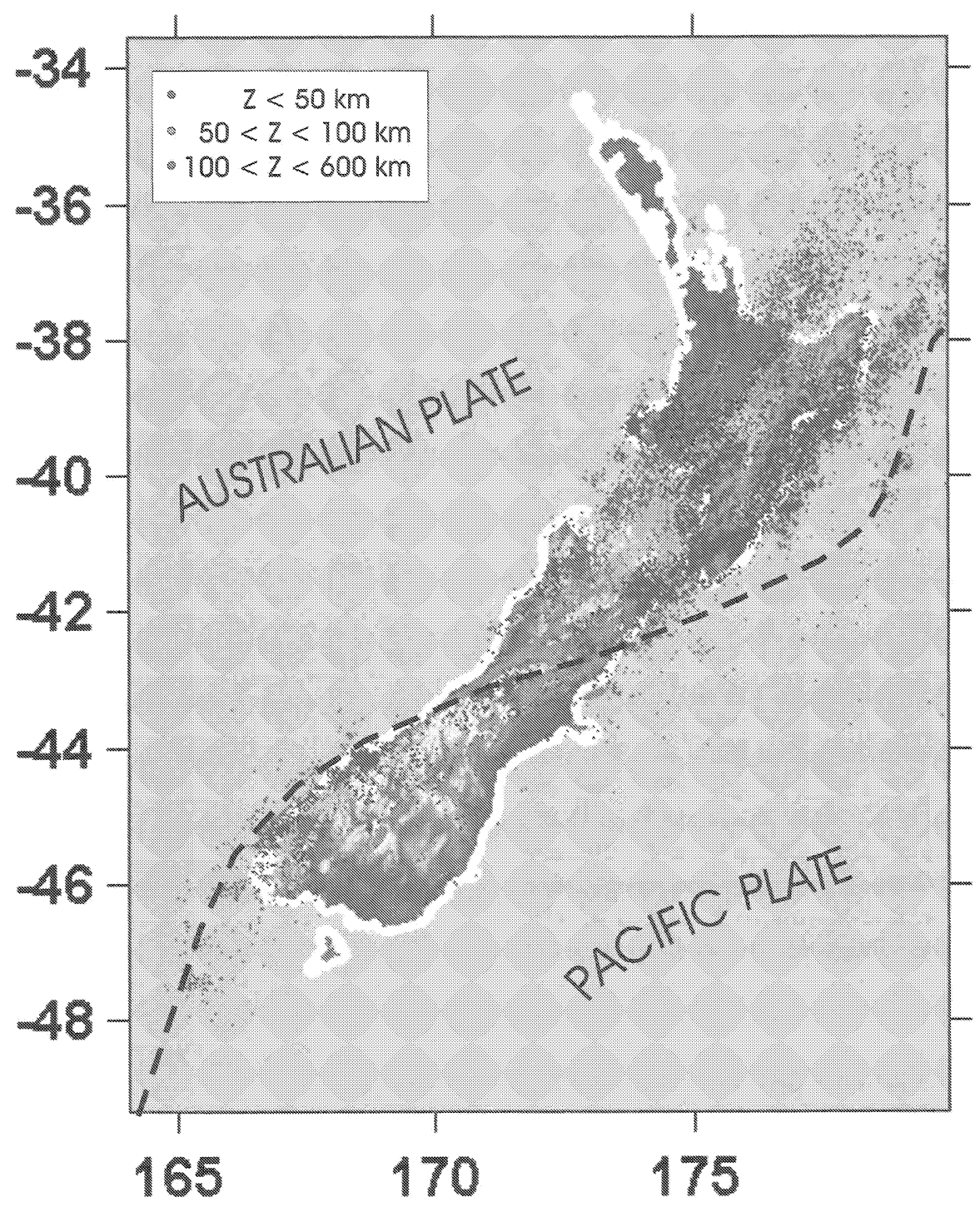

Figure 2. Hypocenters of events occurred from 2001 to 2003 colour coded for depth. The nain tectonic features are also indicated.

The seismic record of a region can be compared to the record of a person's heart beat. The latter gives important information about that person's health state and probable reasons for his or her illnesses, if such is the case. A seismic record also provides key data for interpreting the history of the stress state of a region and may thus give indications when such state departs from a "nomal" state. However, as the previous health history of a person is necessary for reliable diagnostics, in order to make reliable inferences on the seismicity it is imperative to know the changes involved in the seismographic nework's history, which provided the observations in the first place.

A seismic record is a collection of data including date, time, location, size, and, whenever possible, detailed source mechanism characteristics and effects. Such a record is compled in the form of a seismic catalogue. It is a fair assumption to state that the quality of data in the catalogue will be dependant on the quality of the seismograph network used to determine the earthquake parameters mentioned above. It is also appealing to make the statement that a network quality is usually improved over time. However, experience has shown that making this assumption is not always wise. For example, Habemann (1982) demonstrated that the overall teleseismic detection capability in North America was significantly diminished after the closure of the VELA array in the late sixties. He also was able to demonstrate other such changes in reporting for most of the western hemisphere that took place in the mid seventies although the causes were not so clear. 
A precise and updated account of the way earthquake parameters have been reported through the years is difficult to attain as is the chronology of the changes that the network has experienced. If one neglects these characteristics, however, important biases may be introduced in subsequent analyses of the data. For example, variations in the determination of magnitude can be introduced which are related to changes in the routine of estimating earthquake parameters or changes in instrumentation of the networks which provided the raw data. Thus, changes in the number of events per unit time for certain magnitude bins can be due to instrumental and/or operational changes. This can have serious effects on seismic hazard estimates based on seismicity rates for certain areas. Alternatively, knowing the procedures to which certain earthquake data have been subjected may provide enough information as to be able to merge different data sets in a single catalogue and thus increase the amount and quality of data. It can also provide a footing for suitable corrections to the data which may improve the estimates.

The problem of finding artificial variations in seismicity catalogues has been undertaken in various studies (e.g. Habermann, 1982; 1983; Habermann and Wyss, 1984; Wyss and Burford, 1985; Wyss, 1991; Zúñiga, 1989, Zúñiga and Wyss, 1995, Zúñiga et al., 2000). Zúñiga and Wyss (1995) give a brief summary of some important causes for seismicity variations. In what follows we employ techniques described in some of the aforementioned papers as well as in other studies in order to gain insight into the seismicity record of New Zealand.

\section{MAIN CHARACTERISTICS OF CATALOGUE DATA THROUGH TIME}

The main coverage of the catalogue is portrayed in Figure 1 where all events from 1903 to beginning of 2004 are shown. Magnitude scale is $M_{L}$ for most of the events which occurred after 1940, before that date not all events list $M_{L}$ and the catalogue lists other scales as the main reference. Figure 2 shows the main depth characteristics of the data by means of a sample of two years so as not to obscure other features.

In this paper we first analyze the general temporal characteristics of the complete data set and further on we will discuss features which may be dependent on depth. We start with an analysis of cumulative number of events Vs. time graphs as an indicator of variations in trends of the seismicity rate. Figure 3 shows such a plot for all events in the catalogue with or without an assigned magnitude and for two magnitude cutoffs. It is immediately apparent that important reporting changes have taken place with the change which occurred some time in the 1980's dominating the trend. In the Figure we also make a distinction between all events reported (Figure $3 a$ ) as compared to those located south of latitude $\mathrm{S} 34^{\circ}$ (Figure $3 \mathrm{~b}$ ). We can see that the general features remain the same except for the largest events $(M \geq$
7.0). The large events in Fig. 3a which do not show on Fig. $3 \mathrm{~b}$ are located in the Kermadec region and are usually not included in studies dealing with New Zealand seismicity although they are included in the catalogue. Figure 4 shows histograms for the magnitude ranges selected before where we can see that a significant improvement in the amount of data processed took place starting in 1987. The histogram for
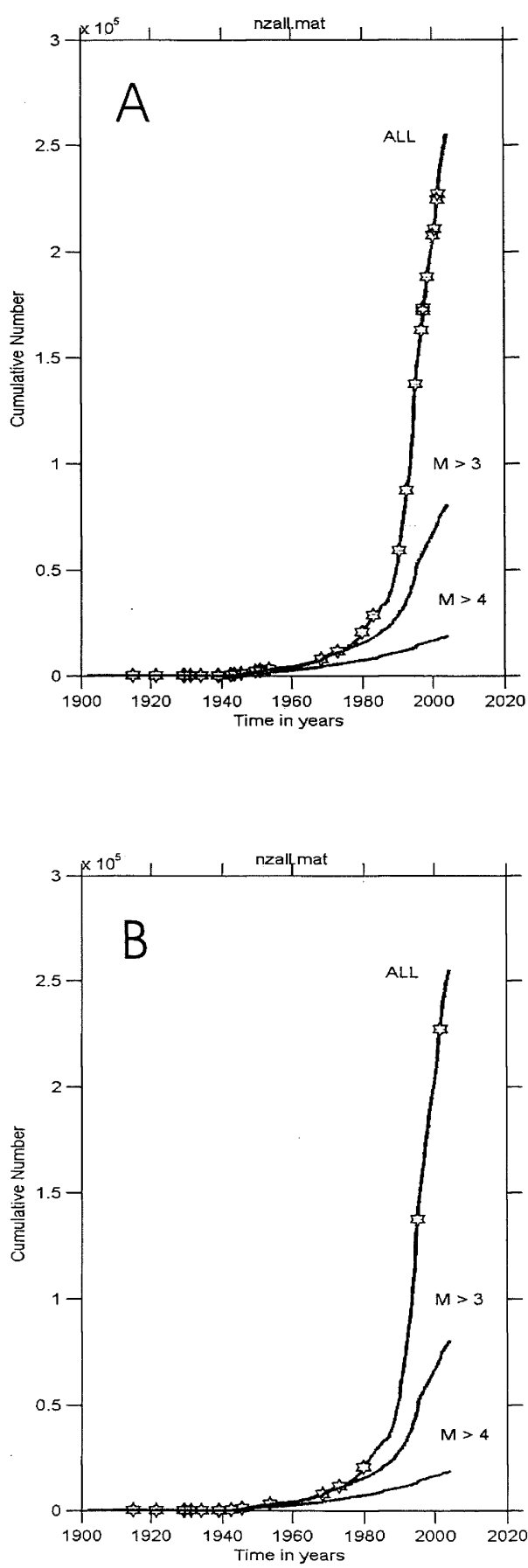

Figure 3. Cumulative number of events Vs. time plots. Stars show events with $M \geq 7.0$. Curves shown are for a) All events in the catalogue, and $b$ ) Events located south of Latitude $\mathrm{S34^{ \circ }}$. 

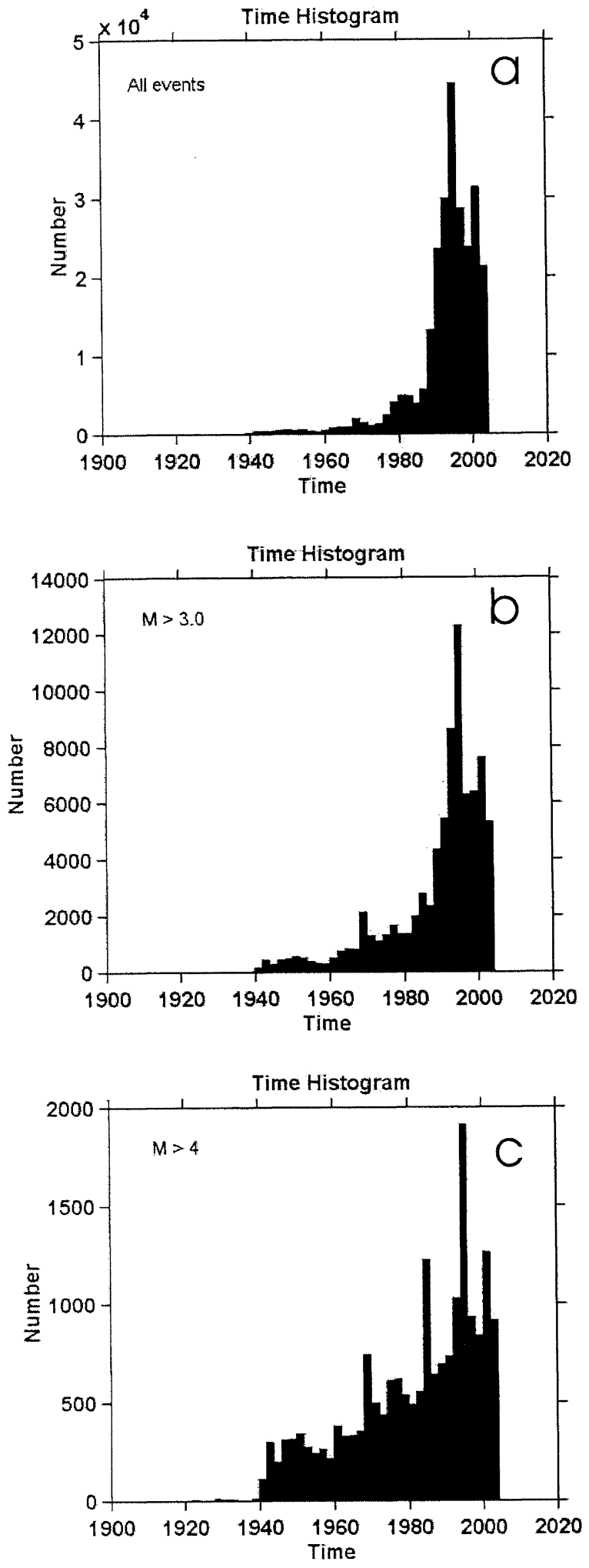

Figure 4. Time histograms for different magnitude cutoffs. a) All magnitudes reported including events with no magnitude assigned, $b$ ) Events with $M>3.0$, c) events with $M>4.0$. events with magnitudes larger than 3 again highlight the same behavior, while that for events larger than 4.0 indicates that a steady increase started earlier, soon after 1940, as already pointed out by McGinty (2001) and Anderson and Webb, (1994) reaching a somewhat uniform level at the end of the 1980's. In Figure 5 we show the cumulative curves for events with magnitudes larger than 5.0 and 6.0 from 1903 to 2003 and Figure 6 shows the corresponding histograms. The data for $M>5.0$ also shows the change which occurred during 1940 but the events with $M>6.0$ do not seem to be affected and a more homogeneous trend is seen from the beginning of the catalogue.

Since the record of magnitudes larger than 4.0 appears to be the one with the longest time span we now turn our attention to details of the cumulative trend of events for that magnitude range from 1940 to 2003 (Fig. 7). We can again see the steady increase in the rate as well as an apparent significant change during the mid 1960's and possibly later, disregarding what appear to be aftershock sequences.

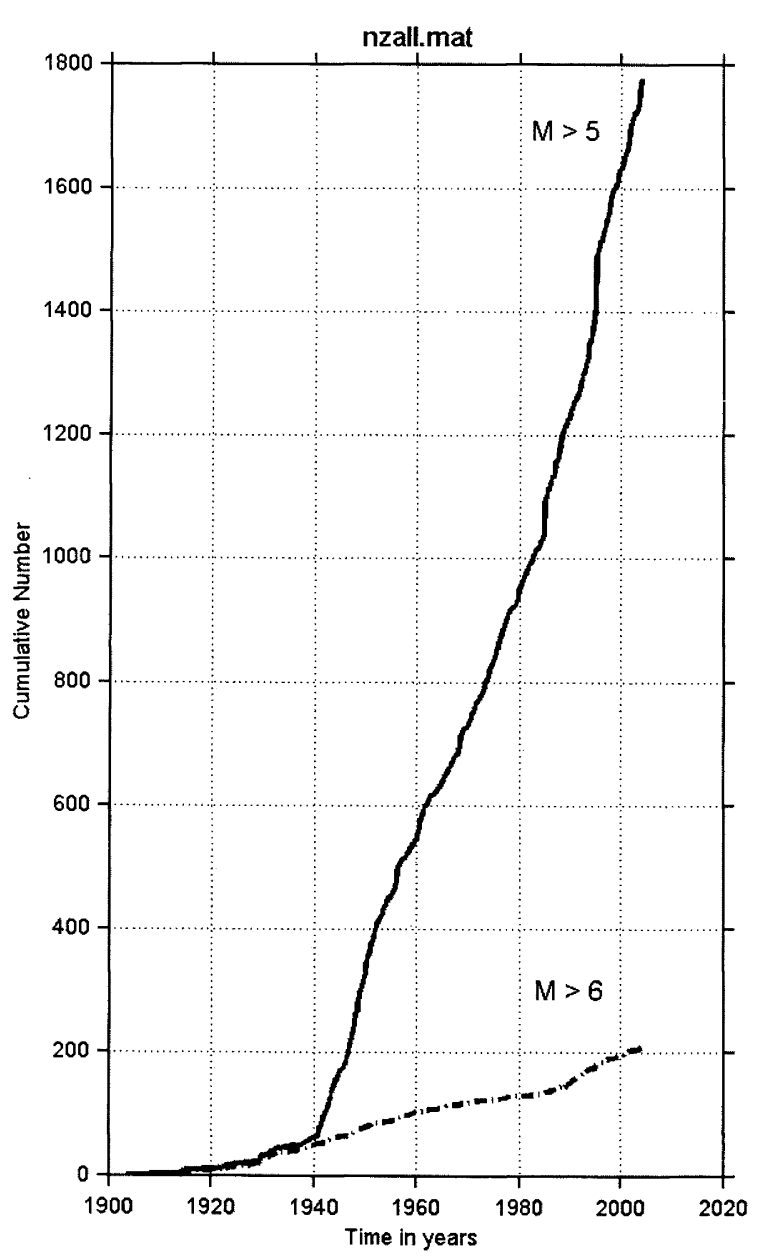

Figure 5. Cumulative number of events Vs time for magnitudes larger than 5 (continuous line) and larger than 6 (broken line). 
In order to pin-point more accurately the time of the most pronounced rate changes for the largest magnitudes, we run algorithm GENAS (Habermann, 1983) to the declustered catalogue within the $M \geq 4.0$ range from 1940 onwards. Declustering is necessary so as not to include aftershock sequences since they incorporate temporary changes which are not related to background rates. Declustering was carried out by means of the procedure of Reasenberg (1985) with the parameters outlined in that study. The cumulative curve for the declustered data set is also shown in Figure 7 where a more constant trend can be observed for the period initiating in the mid sixties even though a subtle change might still be observed at the end of the 1980's. The GENAS algorithm identifies significant changes in seismicity rate (number of events larger and smaller than a given magnitude with respect to time) by comparing the mean rate before the time ( $t$ ) under study to that of the period which follows $t$. This procedure is repeated for increased values of $t$ up to the end of the seismicity record.
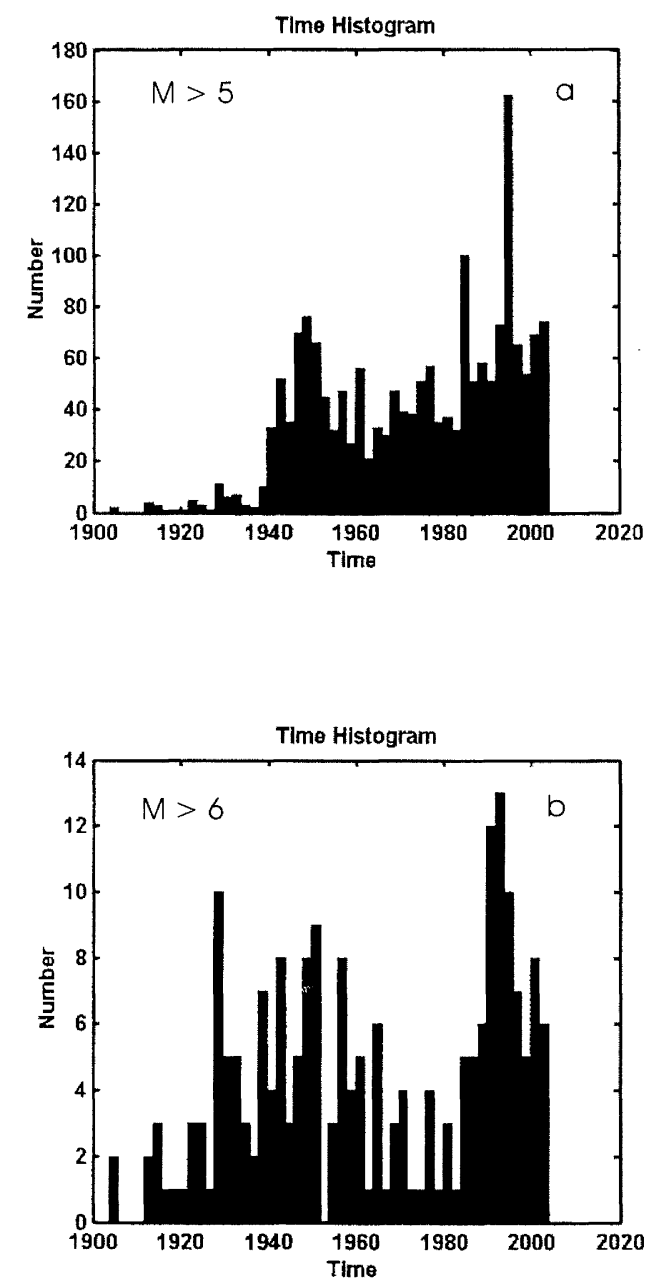

Figure 6. Time histograms for events with magnitudes a) Larger than 5.0 and $b$ ) Larger than 6.0.
Every time a significant change is found, the catalogue is marked and split into two segments which are iteratively analyzed in the same fashion. The algorithm provides the times which stand out as the beginning of periods were increases and/or decreases of seismicity are detected as well as the magnitude range affected by these changes. This tool and others used later in this study have been put together into a software package (ZMAP, Wiemer, 2001; Wiemer and Zúñiga, 1994) which allows a thorough investigation of a seismic record and the seismicity of a region.

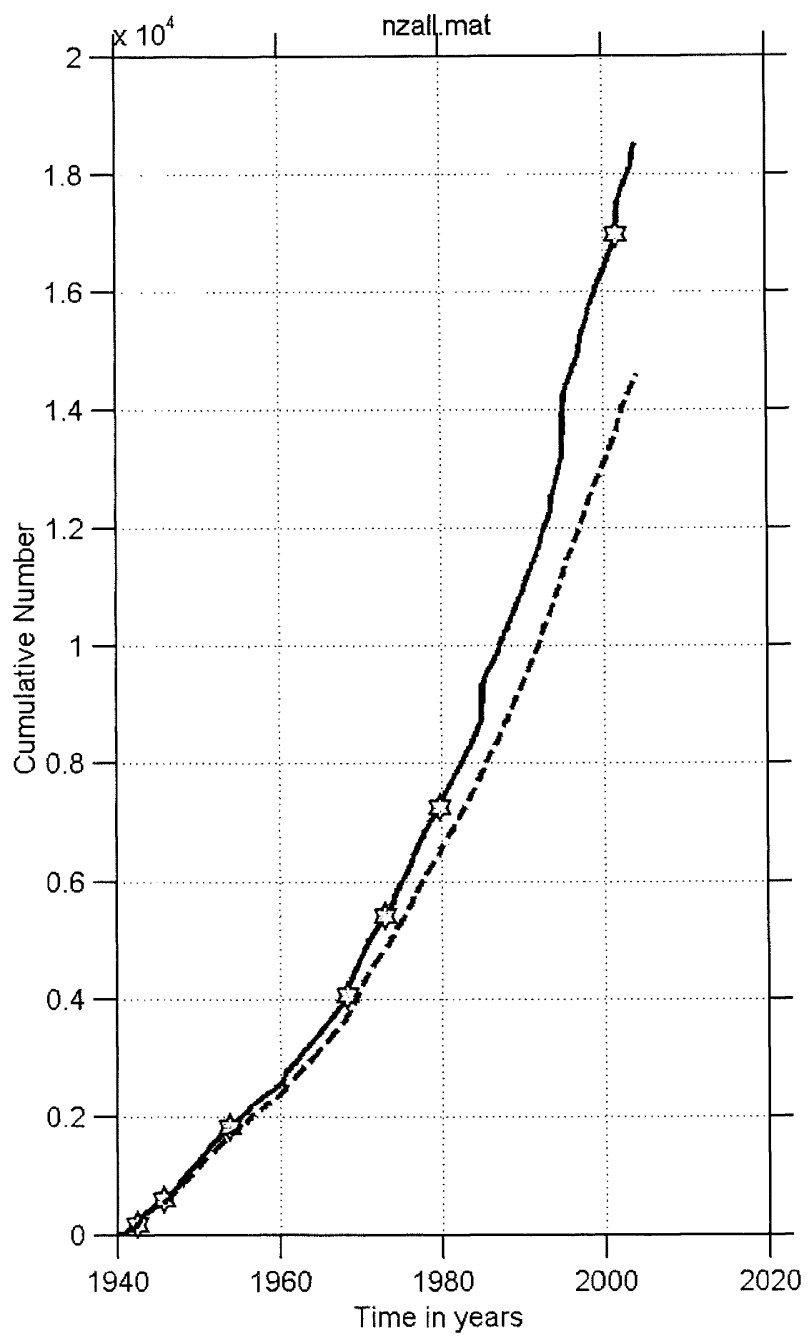

Figure 7. Cumulative number of events Vs time for magnitudes larger than 4 (continuous line) located south of Lat $S 34^{\circ}$ and trend of the declustered data (broken line) from 1940 to 2003. Stars indicate events with $M \geq 7.0$. 
Figure 8 shows the result of algorithm GENAS, where we can now determine that the most significant changes for events with $M \geq 4.0$ reported in the catalogue took place during 1965 to 1968 and in 1987. All changes affected mainly events in the $M<5.0$ range with the sole exception of the change occurring during 1987 (note in particular the right hand panel of Figure 8). Other less significant changes occurred during 1960, 1983 and 1992. The constancy in slope of the cumulative curve shown in Figure 9 for the $M>$ 5.0 segment of the catalogue also indicates that a practically homogeneous level was reached throughout the period, although a subtle rate increase followed by a decrease can be observed occurring during late 1951, also shown on the results of GENAS (Fig. 8).

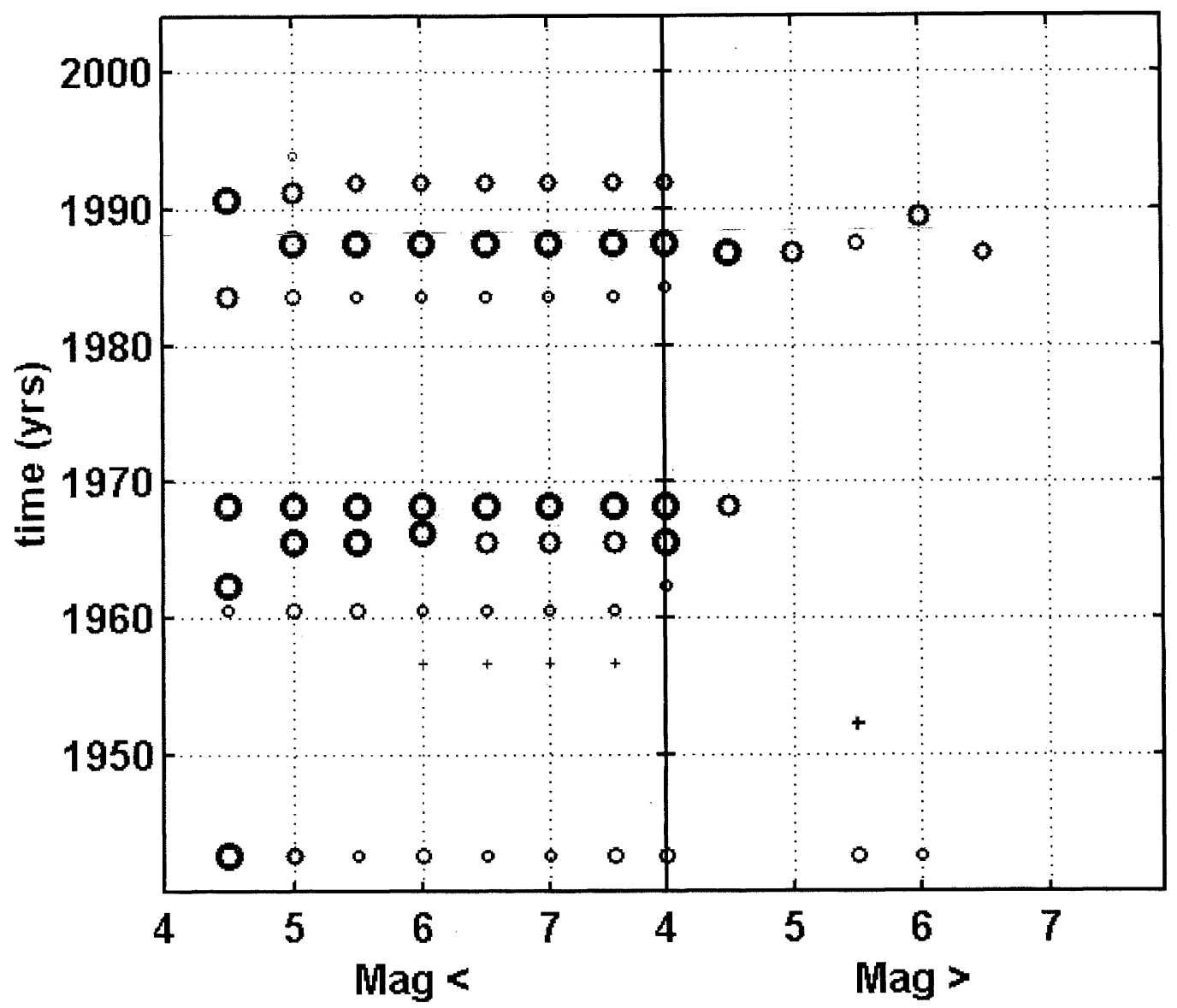

Figure 8. Results of algorithm GENAS for the declustered catalogue from 1940 to 2003 with a magnitude cut-off of 4.0. Only the most statistically significant changes are displayed (larger than 95\% confidence). Circles indicate rate increases, pluses indicate rate decreases. Largest circles indicate confidence of better than $99 \%$.

\section{COMPARISON OF SEISMICITY RATES FOR DIFFERENT TIME INTERVALS}

In order to dwell further into the possible causes of the variations in the complete set of the catalogue we compared the seismicity rates derived from time intervals bounded by the dates found during the earlier stage. For this purpose we can use cumulative frequency-magnitude distributions or FMD (e.g. Gutenberg-Richter curves) as well as noncumulative FMD. The interval from 1965 to 1968 is too short a span for a meaningful comparison, so we merged those dates into a single change. Thus, we looked at the rate from 1940 to 1968 as compared to that from 1968 to 1987 (Fig. 10a). The distributions in both frames of Fig. 10a show that there is a correspondence for both periods starting at magnitude $M=5.3$. From that magnitude on, the similarity between both cumulative distributions is noticeable. This indicates that the $b$ values and annual seismic productivity agree and therefore that a complete magnitude of reporting $\left(M_{c}\right)$ for the interval from 1940 to 1987 for events at all depths would be near $M=5.3$. We notice, nevertheless, that there is an apparent lack of events during the most recent period in the $5.7 \leq \mathrm{M} \leq 6.8$ range, which may be due to the difference in duration between both intervals, since the second interval is about $30 \%$ shorter than the previous.

The period 1968 to 1987 as compared with 1987 to 2004 (Fig. 10b), however, shows a different story. In this case 
there is a difference in the $b$-values and annual productivity which is noticeable at all magnitudes. Since the change is affecting the whole range of magnitudes, and given that we can assume that a good level of completeness for the larger magnitudes $(M>5.0)$ may have already been reached for the later period we can interpret the difference as probably due to changes in instrumentation that took place in the 1980's (Anderson and Webb, 1993). Such changes inadvertently could affect the determination of magnitudes as has been observed to take place in other data sets from various regions around the world (e.g. Zúñiga and Wyss, 1995).

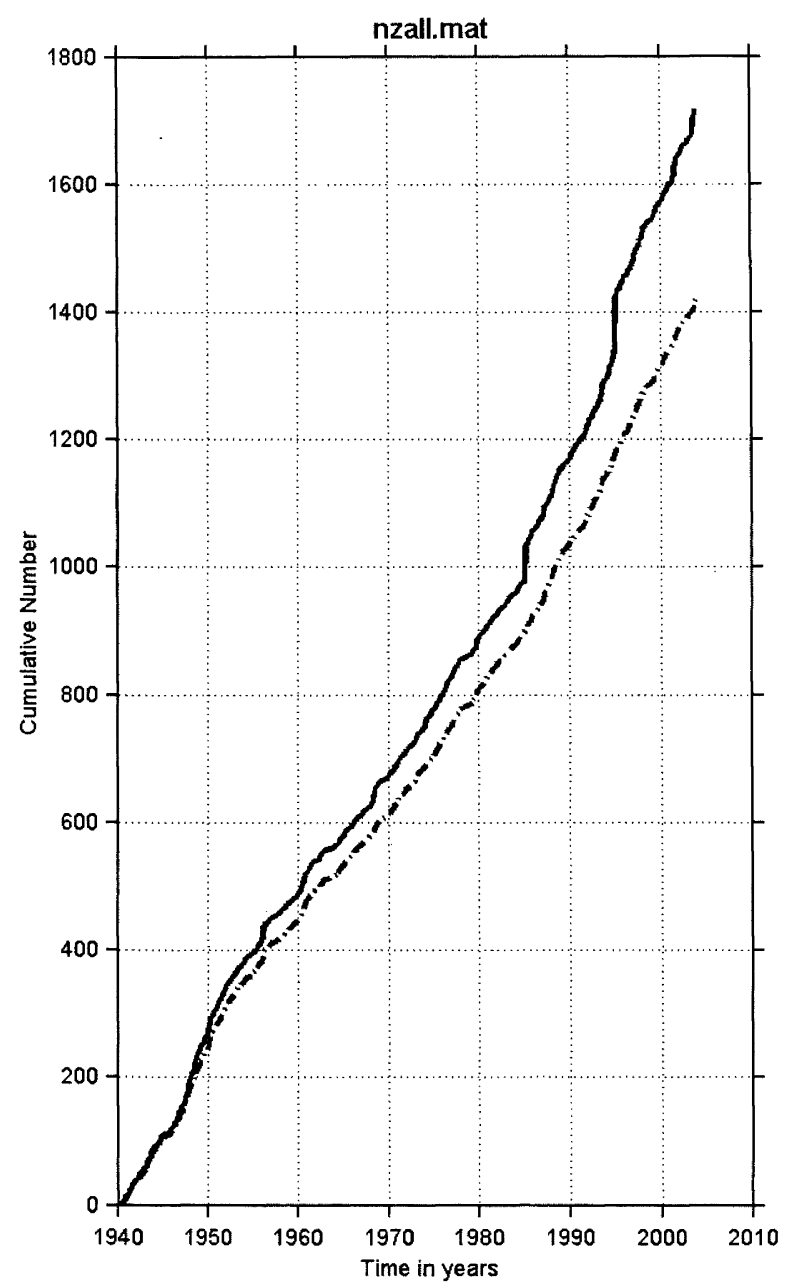

Figure 9. Cumulative number of events Vs time for magnitudes larger than 5.0, original data is shown as a continuous line and declustered data as a broken line.

Thus, in case there was a need to use the whole catalogue for specific objectives, such as global estimates of seismicity, or to calculate general trends to be used as background seismicity, it would be desirable to match the distributions for the two consecutive time intervals. Assuming that a change in magnitude accounts for the variation in rates, we can find a correction to magnitudes in the former interval which best fits the $b$-value distribution of the period we consider most reliable. This procedure, which is also known as $b$-value fitting (Zúñiga and Wyss, 1995), yields the following transformation:

$$
\mathrm{M}_{87-04}=1.11 \mathrm{M}_{68-87}-0.33
$$

where $\mathrm{M}_{87-04}$ are the resulting magnitudes which provide a best fit to the FMD of the period 1987 to 2004 , and $\mathrm{M}_{68-87}$ are the original magnitudes of events in the 1968 to 1987 time interval to which we apply the correction. Figure 10c shows the result of the above transformation where we can see that a very good fit to the current trend is obtained.

\section{DEPTH CHARACTERISTICS}

In taking decisions regarding seismic hazard or when analyzing the tectonic situation of an area, a researcher often needs to differentiate between shallow crustal environments from deeper sources. Furthermore, analysts are usually concerned with the depth of the hypocenter since sometimes other estimates are dependent on this value. It is also customary to allow for some fixed depths when residuals given by the first trial in location are too high. Some of the unconstrained data in the seismic catalogue of New Zealand have been fixed in depth as described below. The question of restricted depth events and suggestions to correct their impact in New Zealand data has been treated by McGinty (2001). Here we will limit our analysis to the main differences in trends by separating shallow from intermediate and deep events.

\section{Shallow seismicity}

Figure 11 shows a depth histogram of all events in the $0-$ $200 \mathrm{~km}$ range from 1903 to present. The three most conspicuous peaks correspond to those events whose depth has been restricted because of poorly constrained initial hypocenter locations. These nominally occur at depths of 5 , 12 and $33 \mathrm{~km}$. A smaller peak occurs for those events with an assigned depth of 0 . In order to avoid problems related to fixed depths, we grouped data into three main depth categories. If we look at the seismicity rates for the declustered $\mathrm{M}>4$ data set in the ranges $0<\mathrm{z}<40 \mathrm{~km}, 40<$ $\mathrm{z}<200 \mathrm{~km}$ and $200<\mathrm{z}<600 \mathrm{~km}$ (Figure 12) a conspicuous feature emerges. The trend for the shallow regime $(0<z<40$ $\mathrm{km})$ appears surprisingly constant suggesting that reporting of events with $M>\sim 4$ has been quite homogeneous since 1940 and implying that the changes discussed previously may affect only the deeper events. Assuming that current conditions allow for complete reporting of $M>4$ events, this observation would suggest that reporting of $M>4$ events in the $0-40 \mathrm{~km}$ depth range has also been close to complete since 1940 
Since, as expected, station coverage was far from current standards in those earlier dates it was necessary to verify the validity of the above observation. Therefore, in order to obtain a more accurate estimate of the completeness magnitude for shallow events we compared FMD's for the interval 1940 to 1968 with that for 1968 to 1987 . Figure 13a, as before, shows cumulative and non-cumulative magnitude distributions for the mentioned intervals. It can be seen that the curves for both intervals match each other well starting at $\mathrm{M} \sim 4.5$ which suggests that such value may be close to the actual minimum magnitude of completeness for 1940 onwards. Analyzing the trend from 1968 to 1987 as compared to that of 1987 to 2004 (Fig. 13b) we can observe again a good correlation between curves but in this case starting at $\mathrm{M} \sim 3.9$ indicating that $M_{c}$ was lowered close to such a value. It is also noteworthy that the change in $b$-value observed for the whole of the catalogue is not seen at shallow depths. As already mentioned, the spatial characteristics of the catalogue, including $M_{c}$, are discussed in a second paper.
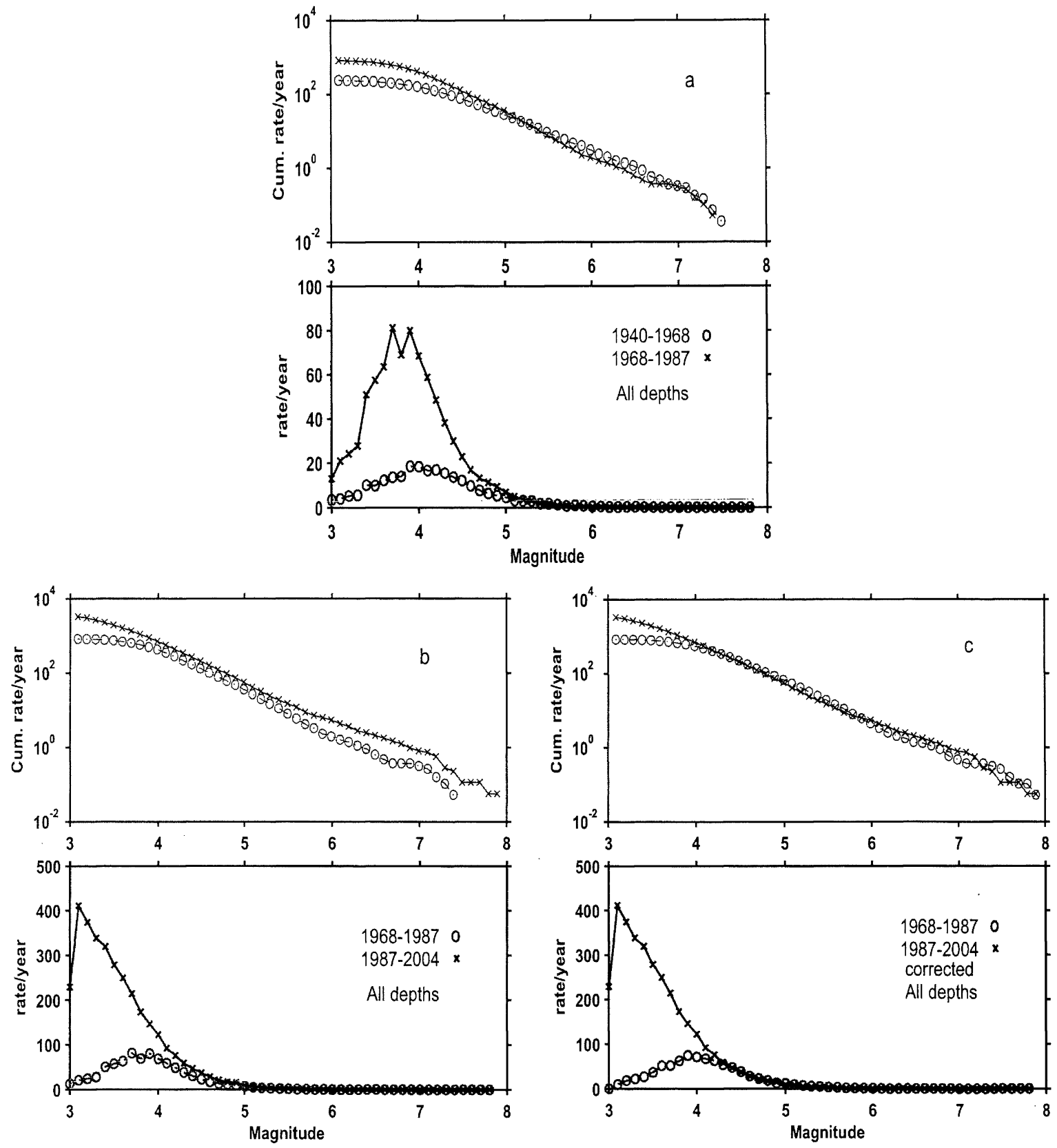

Figure 10. Comparison of cumulative (upper frame of each panel) and noncumulative (lower frame) Frequency-Magnitude distributions derived from two consecutive time intervals for the whole data set. a) Magnitude data from 1940 to 1968 as compared to 1968 to 1987. b) Magnitude data from 1968 to 1987 compared to 1987 to 2004. c) Same periods as in b but after applying a correction to the data in the first interval (see text). 


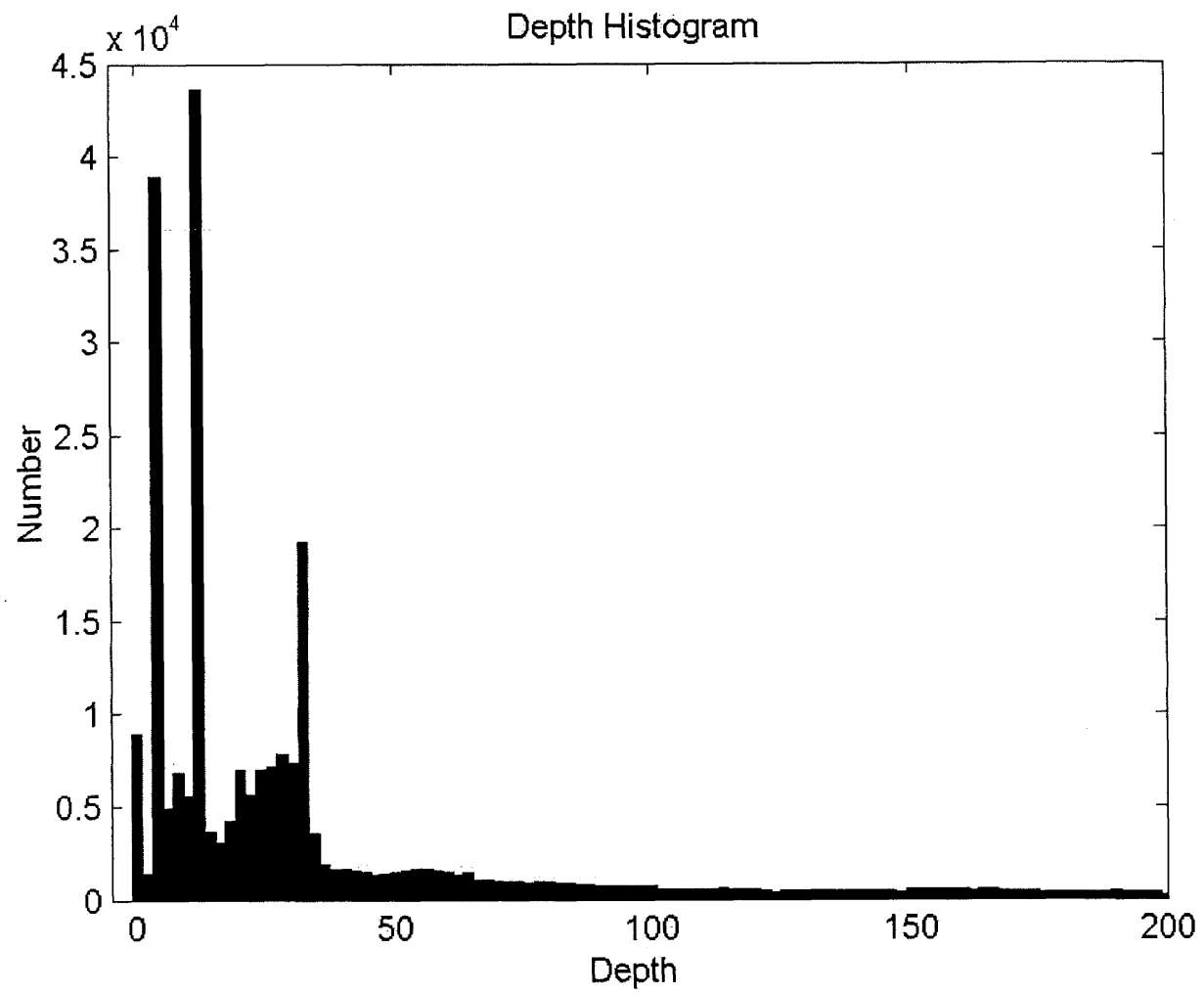

Figure 11. Depth distribution of events from 1903 to 2004 in the range 0-200 km.

Since these $M_{r}$ values appeared somewhat too low, in light of the station coverage of the earlier dates, we carried out a second analysis on that data subset in order to provide additional support for these observations. For this purpose, we employed the technique proposed by Wiemer and Wyss (2000), which estimates $M_{c}$ by a calculation of differences between the observed FMD and a synthetic distribution based on the $b$ - and $a$-value of the Gutenberg Richter law which represents a perfect fit to a power law. To estimate the goodness of the fit we computed the absolute difference, or residual, $R$, of the number of events in each magnitude bin between the observed and synthetic distribution. Figure 14 shows plots of residuals as a function of $M_{c}$ for the three time intervals discussed above. The minimum residual provides an estimate for the best fit between the observed and the synthetic FMD and the minimum magnitude at which the residual is obtained therefore can be used to obtain an alternative measure of $M_{c}$. By considering the minimum in the graph of Fig. 14a we can estimate that from 1940 to 1968 , $M_{r}$ reached a value of 4.4 within a confidence of $90 \%$. If we now look at the residuals for the interval 1968 to 1987 (Fig. $14 \mathrm{~b}$ ) it is apparent that a minimum plateau is reached starting at a $M_{c}$ value of 3.9. Finally, Fig. $14 \mathrm{c}$ shows residuals for the latest interval (1987 to 2004) where we can estimate $M_{c} \approx$ 2.6.

\section{Intermediate and deep seismicity}

We now turn our attention to the deeper events by making a comparison between the rate for 1940 to 1968 as compared to that of 1968 to 1987 for events in the $40<\mathrm{z}<600 \mathrm{~km}$ depth range. Analyzing the curves in Fig. 15a we can observe basically the same characteristics as when considering the whole depth range (Fig. 10a). That is, there is a slight difference in $b$-value which might be taken as indication of changes in magnitudes due to instrumental variations, contrary to what was observed for the shallow part of the catalogue. Another feature which is noticeable from the figure, is that both FMD start matching at a magnitude $\mathrm{M}$ 5.0 which is higher than the magnitude at which the distributions matched for the shallow events. Applying the $b$ value fitting technique, we found that a magnitude transformation which allows for the FMD of the 1940 to 1968 interval to match the one for the 1968 to 1987 interval is the following:

$$
\mathrm{M}_{68-87}=0.72 \mathrm{M}_{40-68}+1.48
$$

If we apply such magnitude transformation the FMD's match each other starting at a magnitude of approximately 4.6 (Fig. $10 \mathrm{~b})$ 
The comparison between the rate for 1968 to 1987 with that of 1987 to 2004 for the intermediate and deep events (Fig. 15c) also shows basically the same features as the comparison for the whole range of depths (Fig. 10b), that is, an apparent lack of correlation at most magnitude bands. The similarity between these curves and the ones seen for the complete data set indicate that deep events dominate the trends observed previously. Nevertheless, by the manner in which the two FMD depart we are faced with two possible corrections. The first alternative is to apply the $b$-value fitting technique (also called a magnitude "stretch") in order to match the FMD of the most recent interval. This yielded the following magnitude transformation:

$$
\mathrm{M}_{87-04}=1.23 \mathrm{M}_{68-87}-0.84
$$

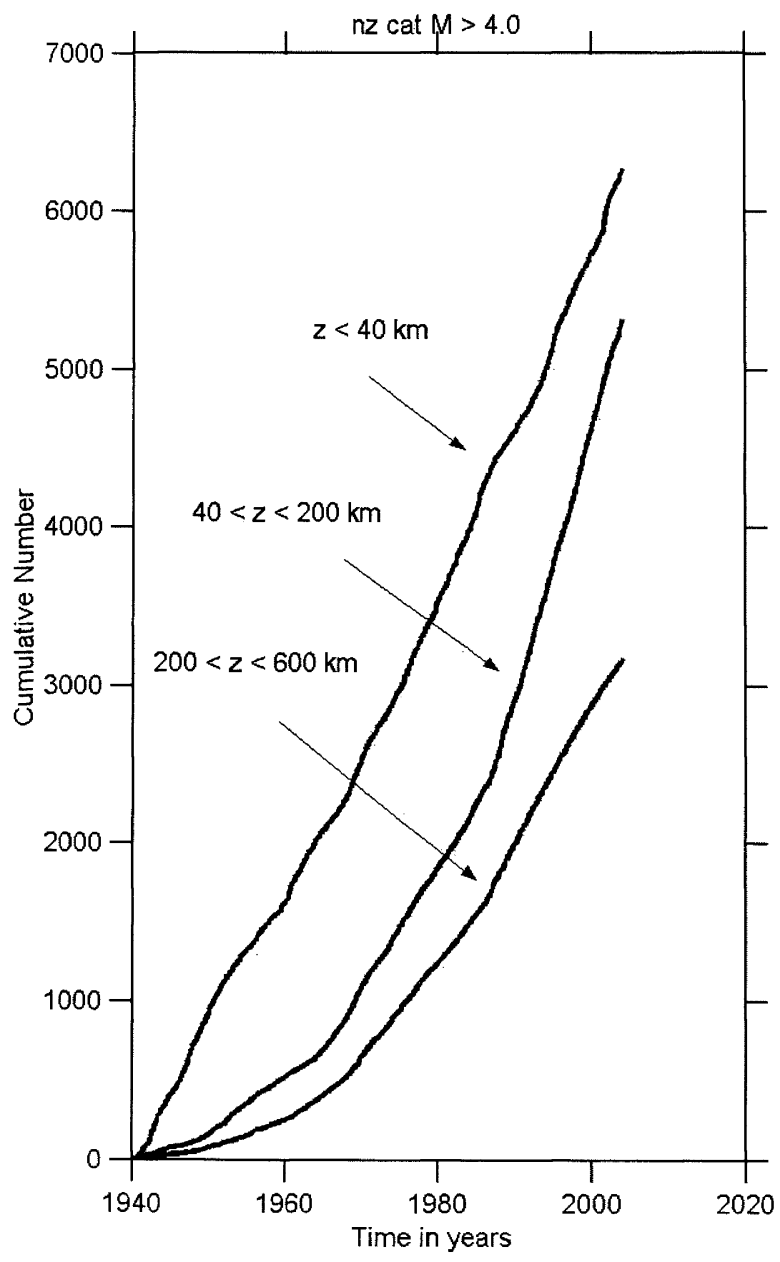

Figure 12. Cumulative number of events Vs. time for the $M>4.0$ declustered set at three distinct depth ranges.
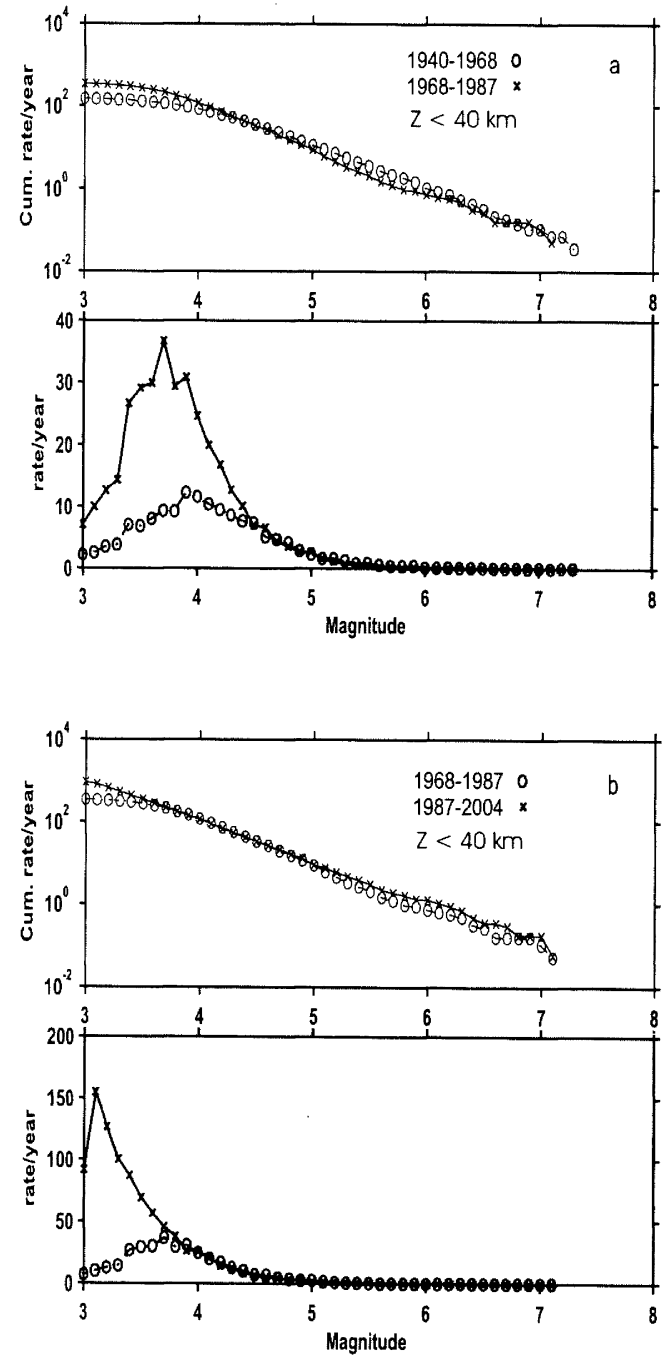

Figure 13. Comparison of cumulative and noncumulative Frequency-Magnitude distributions from two consecutive time intervals for events in the $z<$ $40 \mathrm{~km}$ depth range. a) Magnitude data from 1940 to 1968 as compared to 1968 to $1987 . b$ ) Magnitude data from 1968 to 1987 compared to 1987 to 2004.

The results of this correction are shown in Fig. $15 \mathrm{~d}$ where it can be seen that the correction provides a good fit to the cumulative distribution of the current trend. However, it has to be noted that this correction effectively shifts magnitudes by over 0.5 units for the largest magnitudes $(M>5.9)$ which is not an optimum outcome. The second alternative is to 
employ a simple magnitude shift of 0.2 units. The match between the magnitude shifted data for the interval 1968 to 1987 and the uncorrected data for 1987 to 2004 is shown in Figure 15e. In this case we obtain a good fit between both cumulative distributions at the lower magnitude range $(M<$ $5.5)$ but there is a noticeable difference for larger magnitudes. The apparent drift for larger magnitudes starting at $\mathrm{M} \sim 5.5$ can be due to the actual fluctuations in number of large events for these intervals since earthquake productivity at this level is of the order of one event/year. Due to the fact that the last correction has a smaller effect on the data we would be inclined towards its usage. Both of these corrections can be useful if one needs to incorporate data for the period 1968 to 1987 in order to make more precise $b$ value estimates and comparisons.
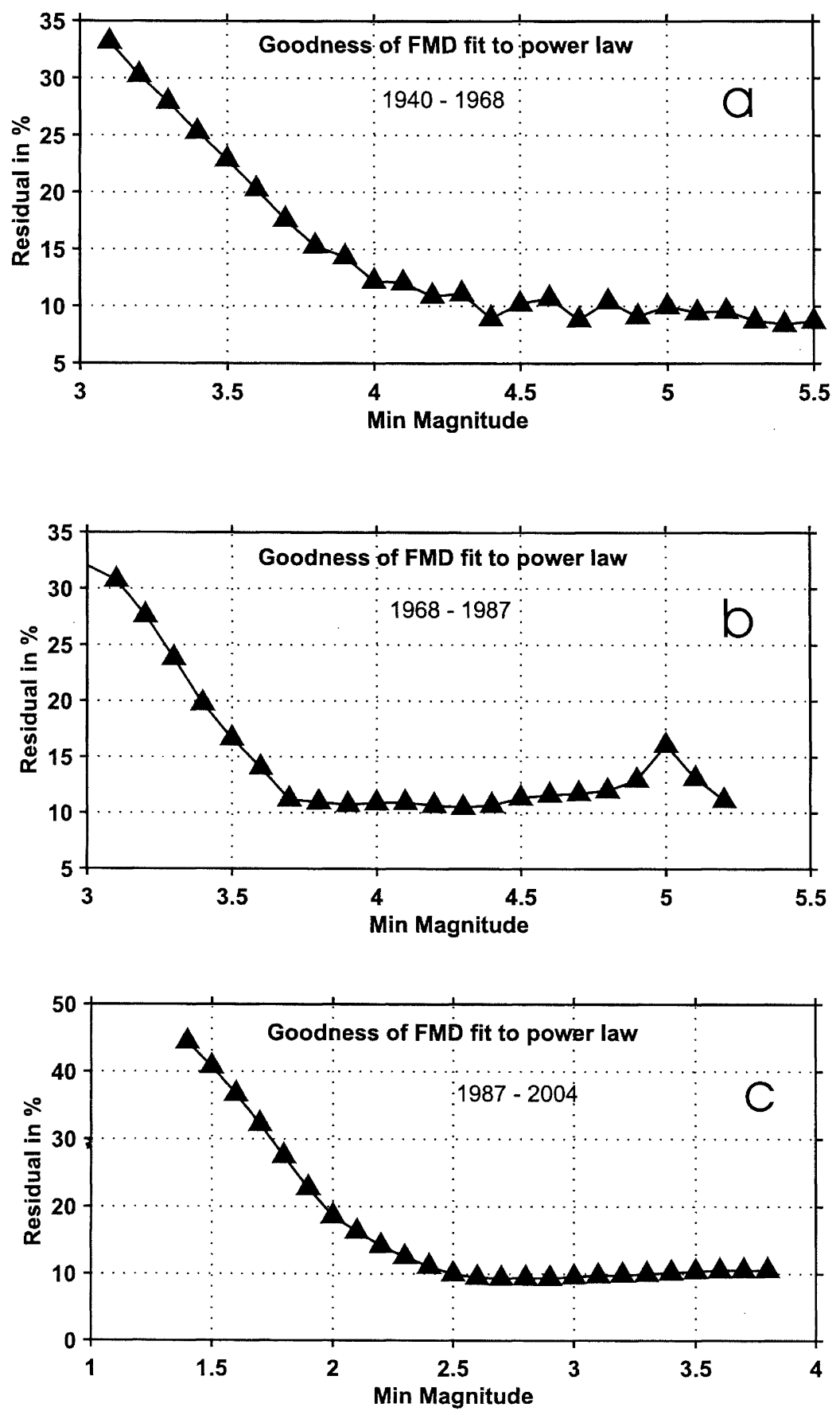

Figure 14. Goodness of fit of FMD to power law as a function of different $M_{c}$ cutoffs for shallow data. a) Data from the interval 1940 - 1968. b) Data from the interval 1968 - 1987. c) Data from the interval 1987-2004. 

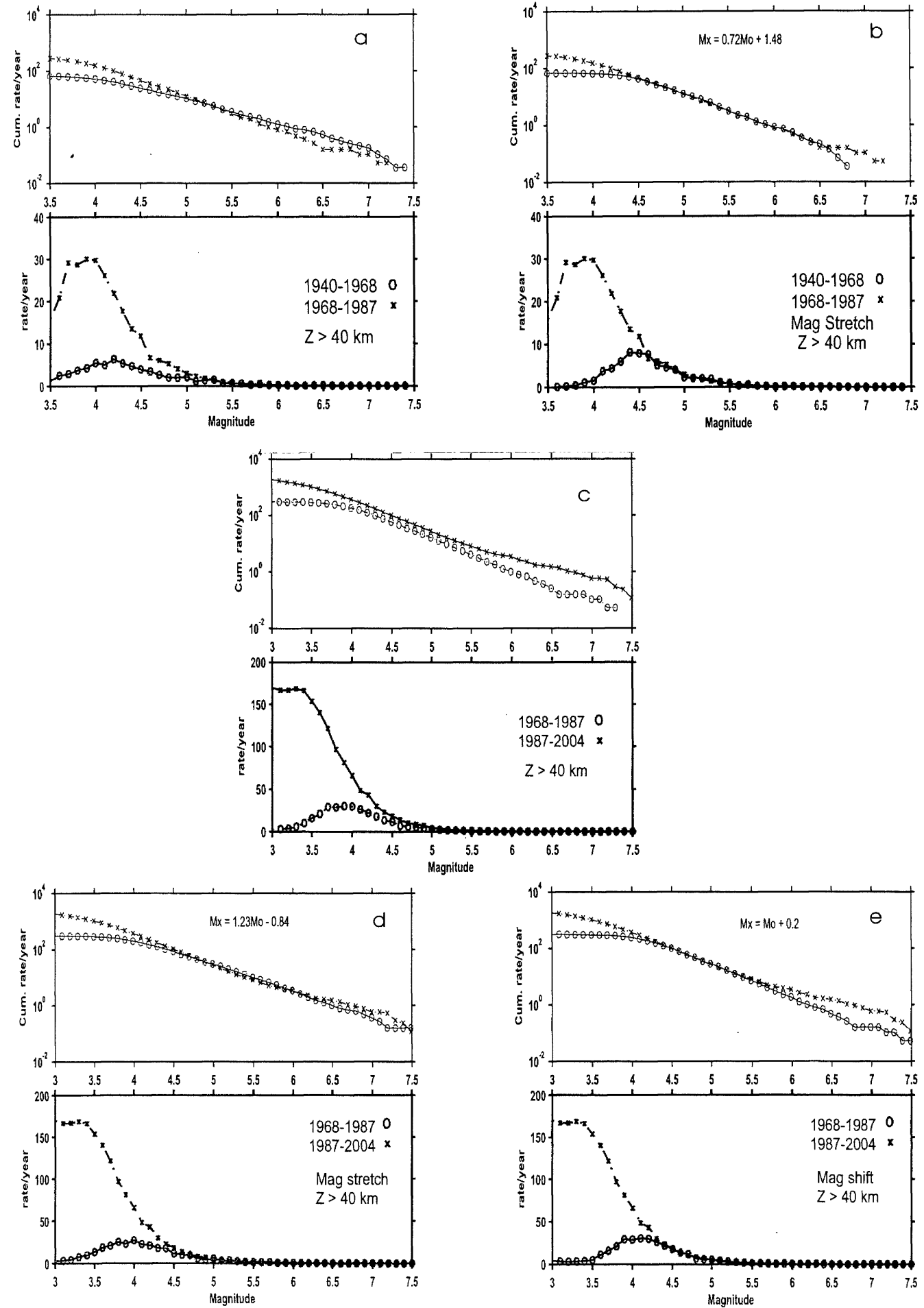

Figure 15. Comparison of cumulative and noncumulative Frequency-Magnitude distributions from two consecutive time intervals for events in the $40<z<600 \mathrm{~km}$ depth range. a) Magnitude data from 1940 to 1968 as compared to 1968 to 1987. b) Same as in a, but after applying a linear correction to magnitudes in the former interval. c) Magnitude data from 1968 to 1987 as compared to 1987 to 2004. d) Same as in c but after applying a linear correction to magnitudes in the former interval. e) Same as in $c$ but after applying a simple shift to magnitudes in the former interval. 
As was done in the case of the shallow data, we performed an additional estimate of $M_{c}$ for intermediate and deep events using the procedure outlined previously. In Figure 16a we plot residuals between observed and synthetic FMD's vs. $M_{c}$ for intermediate and deep data occurring between 1940 and 1968. The minimum is observed to take place at $M_{c} \approx 5.5$ even though the residuals appear to reach a plateau at a $M_{c}$ value of approximately 4.2 . It is conceivable that $M_{c} \approx 4.2$ might be the actual minimum magnitude due to the simplicity in waveforms which result because of efficient propagation of seismic waves up the subducted slab to eastern North Island stations. The results for the time 1968 to 1987 (Fig. 16b) are markedly different in that the difference in residuals between the minimum (at approximately $M_{c}=5.4$ ) and the start of the plateau, at approximately $M_{c}=4.0$, is not significant so $M_{c}=4.0$ could be considered the minimum magnitude for this period. Finally, residuals for the interval 1987 to 2004 (Fig. 16c) indicate that $M_{c}=3.6$ is a suitable estimate for the current trend.
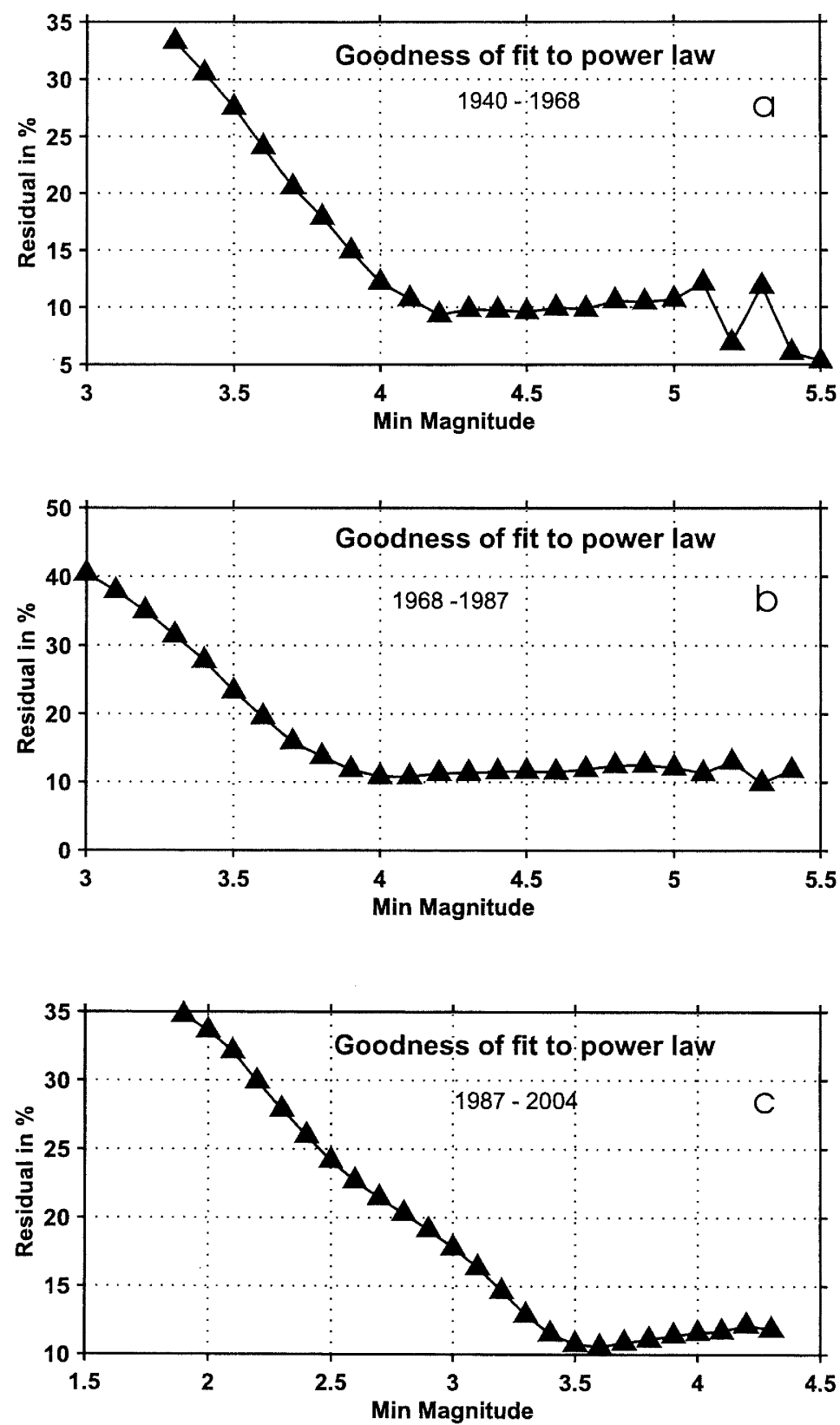

Figure 16. Goodness of fit of FMD to power law as a function of different $M_{c}$ cutoffs for intermediate and deep data. a) Data from the interval 1940 - 1968. b) Data from the interval 1968 - 1987. c) Data from the interval 1987-2004. 


\section{VARIATION OF MAGNITUDE OF COMPLETENESS WITH TIME}

The minimum magnitude of completeness is an important parameter for seismicity and hazard studies, since it is necessary to use the largest possible number of events which reliably represent the activity in order to make useful inferences. If a single overall $M_{c}$ cutoff that is high is employed in order to guarantee completeness, we might be throwing away useful data which in some cases may be crucial.

It is no surprise that $M_{\mathrm{c}}$ changes in time (usually decreasing) since it is dependent on the quality and quantity of stations that comprise a network which ideally improve over time. Few studies, however, report the time variation of $M_{\mathrm{c}}$. Such variations may play an important role in the determination of seismic hazard, in particular when mixing data from different times and/or magnitude ranges together.

We looked at $M_{\mathrm{c}}$ as a function of time for the different time intervals discussed before as well as for the two different depth ranges. $M_{c}$ was calculated as the point of maximum curvature of the non-cumulative distribution which has been observed to be an effective additional way to estimate it and it allows less computing time. A running window with 500 to 1000 events was employed to carry out the estimate. This number was adjusted according to the amount of data available for the interval. The results may differ slightly from estimates carried out using other methods, however, since our objective is to make a comparison among trends at different intervals, we consider that this technique is appropriate because it lacks the uncertainties of other methods.

Figure 17 shows the variation of $M_{\mathrm{c}}$ for shallow $(\mathrm{z}<40 \mathrm{~km}$ ) seismicity in the three time intervals identified previously. We can observe that during the interval 1940 to 1968 (Fig. 17a) $M_{c}$ mostly oscillated between 3.5 and 4.3 . It is interesting to note that a noticeable but short lived improvement of the reporting threshold took place around 1955 with values later on returning to and even getting larger than the previous thresholds. For the interval 1968 to 1987 (Fig. 17b) $M_{\mathrm{c}}$ starts with surprisingly low values close to 3.0 and later oscillates between 3.5 and 4.0 up to around 1978 when the threshold was considerably improved to values of less than 2.0. The low threshold around 1968 is almost certainly due to the two portable stations installed immediately after the Inangahua earthquake of 23 May 1968. These allowed the location of 809 events during their 40 days of recording. After $1978 M_{\mathrm{c}}$ remained at levels close to 2.0 until 1984 when a probable network change increased $M_{\mathrm{c}}$ to pre-1978 values. That average lasted for a couple of years and then values started to decrease again reaching the same average as observed for the time 1978 to 1984 . During the latest interval which runs up to now (Fig. 17c) the trend appears steadier with a mean of $2.3 \pm 0.4$. Even though there have been some instances when the level has reached values of 1.0 and some others when $M c$ has increased back to old averages, these are likely to be due to aftershock or cluster sequences which usually bias the trend.
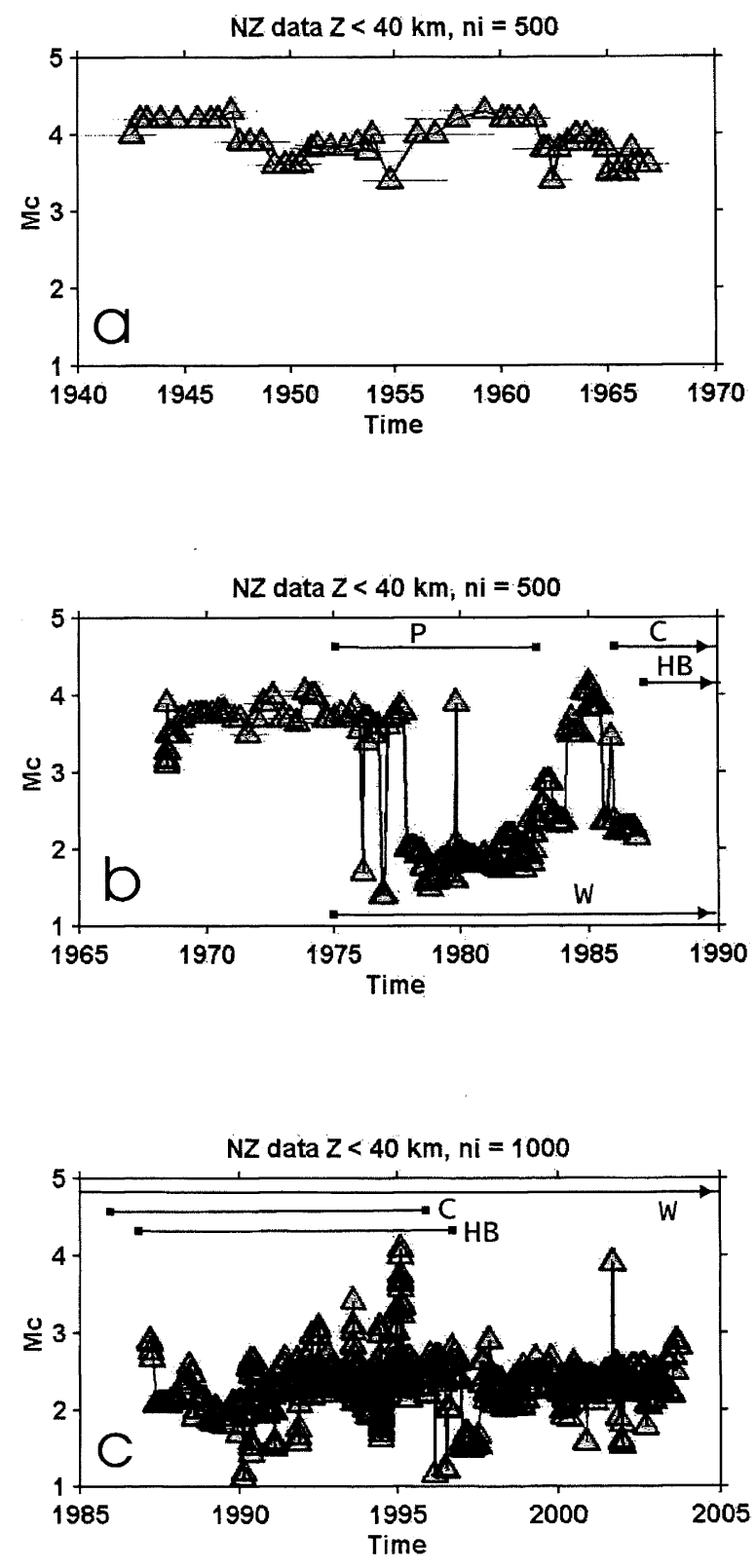

Figure 17. Variation of magnitude of completeness Vs. time for shallow $(Z<40 \mathrm{~km})$ reported seismicity. The number of events employed in the running window is shown on each frame. a) Data in the interval 1940 to 1968; b) Data in the interval 1968 to 1987; c) Data in the interval 1987 to 2004. Horizontal lines outline the duration of installation of microearthquake networks. $C=$ Clyde Network, HB = Hawke's Bay Network, $P$ $=$ Pukaki Network, $W=$ Wellington Network. 
As a further test of the stability of the method, Fig. 18 shows the cumulative and non-cumulative frequency-magnitude distributions for the period 1987 to 2004 with an $M_{\mathrm{c}}$ estimated as the point where the linear fit obtained through weighted least squares departs from the observed data. Using this method we get $M_{c}=3.7$ for $b=1.07 \pm 0.02$ (the maximum likelihood estimate for the same $M_{\mathrm{c}}$ gives $b=$ $0.886 \pm 0.008)$. We notice that there is a previous linear segment which would lower the $M_{\mathrm{c}}$ estimate although it does not include the largest event. The curves in Fig. 18 also show that the $M_{\mathrm{c}}$ estimated by the maximum curvature of the noncumulative distribution in the time variation analysis (approximately $M_{\mathrm{c}}=2.3$ for the last time interval) corresponds to the first linear segment of the distribution, which falls between both $M_{\mathrm{c}}$ estimates.

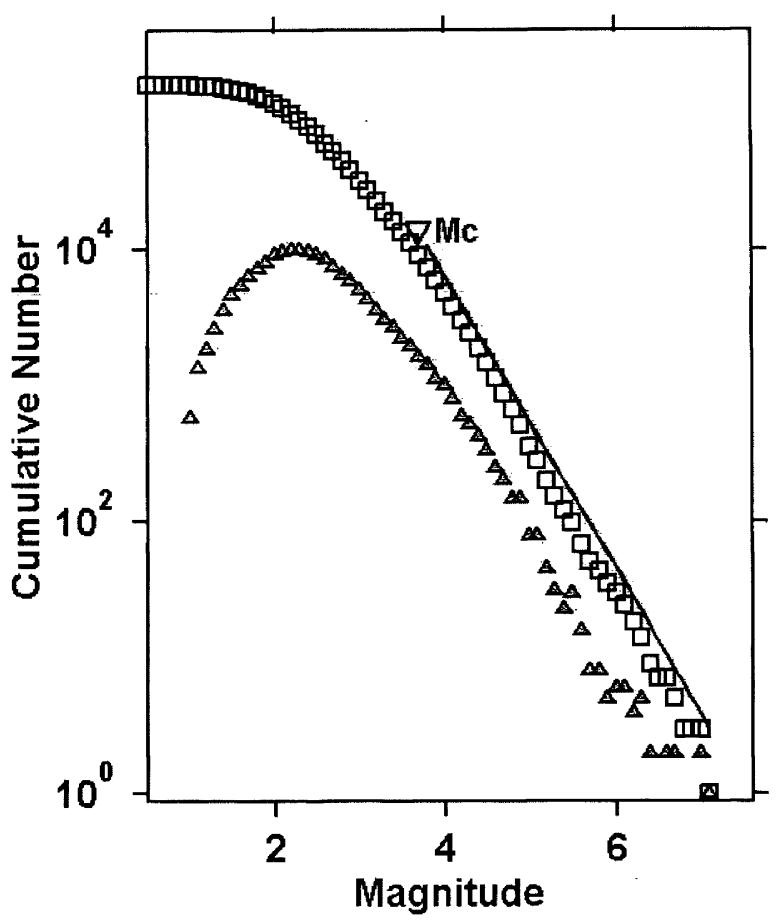

Figure 18. Cumulative (squares) and non-cumulative (triangles) frequency-magnitude distributions for shallow $(Z<40 \mathrm{~km})$ events in the period 1987 to 2004. The line shows the weighted least squares fit $(b=1.07 \pm 0.02) . M_{c}$ is the minimum magnitude of completeness.

The results for intermediate and deep seismicity are again somewhat different than those for the shallow events since for the 1940 to 1968 interval (Fig. 19a) $M_{\mathrm{c}}$ has a mean of 4.2 \pm 0.1 without the fluctuations seen for the shallow counterpart. The interval 1968 to 1987 (Fig. 19b) shows a steady mean of $3.9 \pm 0.1$ up till 1978 , with some short instances, from 1978 to 1982 , when $M_{\mathrm{c}}$ was reduced to values of 2.0 coinciding with the times when the threshold was improved for shallow events. In this case however, as opposed to the shallow case, the threshold returned to the previous larger $M_{\mathrm{c}}$ values around 1982, with a mean of $3.7 \pm$ 0.1 for the latest part of the interval. The last interval (1987 to 2004, Fig. 19c) which includes current standards shows smaller scatter than the shallow data from the same interval (Fig. 17c) and $M_{\mathrm{c}}$ oscillates between 2.7 and 3.3, which is higher than the average observed for the shallow counterpart.
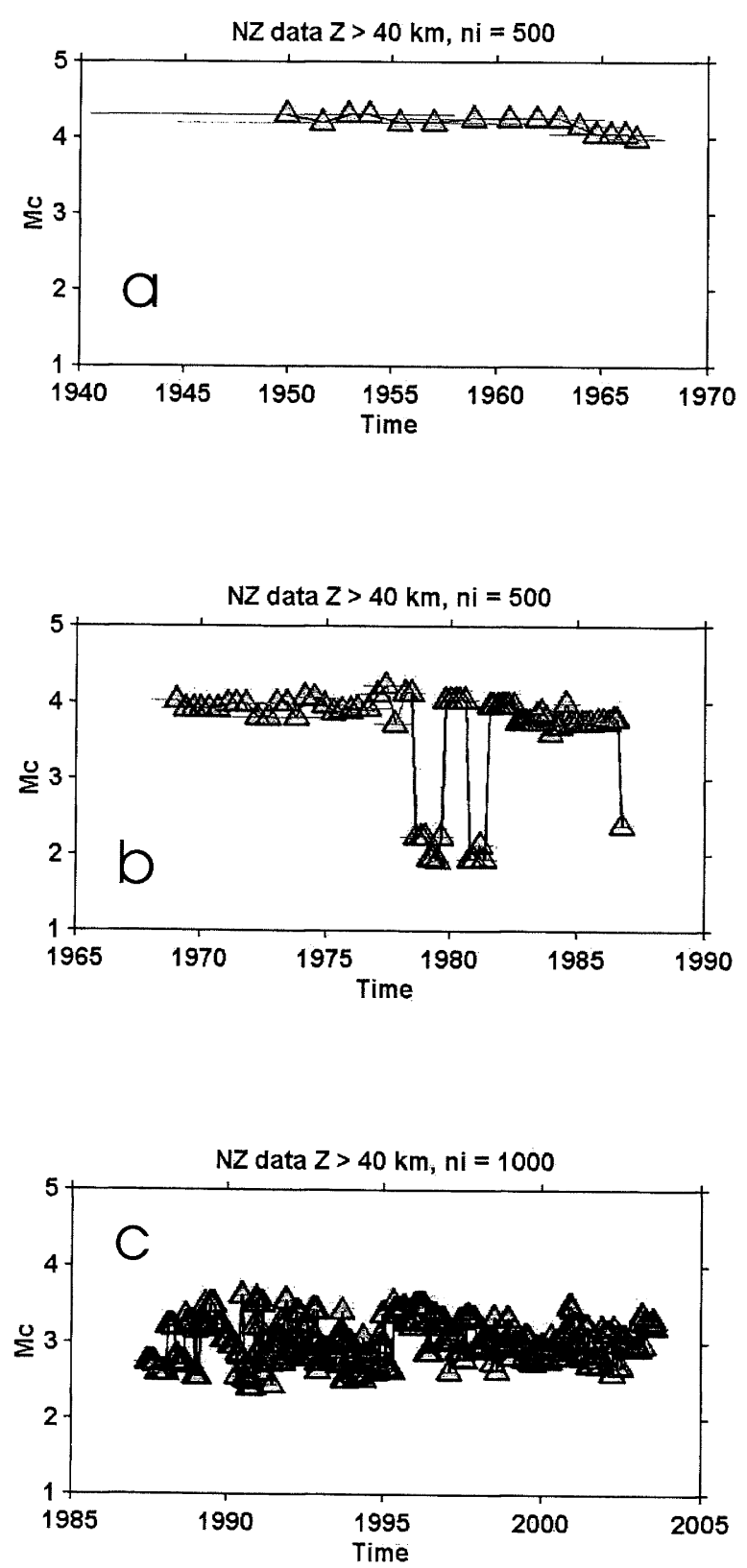

Figure 19. Variation of magnitude of completeness Vs. time for intermediate and deep $(Z>40 \mathrm{~km})$ reported seismicity for the three time intervals of interest. 
Figure 20 shows the cumulative and non-cumulative frequency-magnitude distribution for the intermediate and deep seismicity $(Z>40 \mathrm{~km}$ ) in the period 1987 to 2004 . Using a weighted least squares fit to the longest linear trend we estimate $M_{c}=3.6$ and $b=1.06 \pm 0.02$ (the maximum likelihood estimate for this $M_{\mathrm{c}}$ is $b=0.929 \pm 0.007$ ). As in the previous case, there is a difference between $M_{\mathrm{c}}$ estimated from the maximum curvature of the non-cumulative distribution for the time variation analysis, and the value obtained from the least squares fit. In this case, however, a bi-modal trend is noticeable at the lower end of the magnitude range in the non-cumulative distribution which may be due to spatial variations in $M_{\mathrm{c}}$. Nevertheless, since the difference between this estimate and that of the remains stable, it is possible to calculate one from the other.

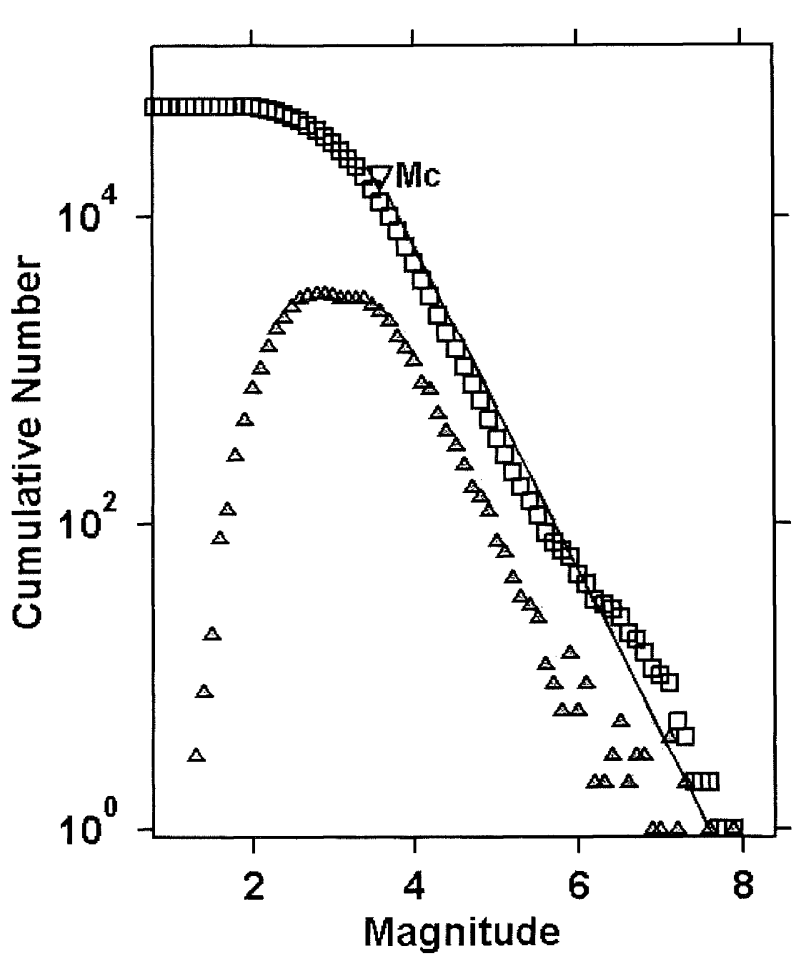

Figure 20. Cumulative (squares) and non-cumulative (triangles) frequency-magnitude distributions for intermediate and deep $(Z>40 \mathrm{~km})$ events in the period 1987 to 2004. The line shows the linear fit $(b=1.06 \pm 0.02)$ obtained from weighted least squares. $M_{c}$ is the minimum magnitude of completeness for this estimate.

\section{DISCUSSION AND CONCLUSION}

The catalogue of seismicity of New Zealand has valuable information dating back to the beginning of the twentieth century. Knowing the dates when major changes have taken place as well as some of the effects on the parameters can help make the best of the data.

The trends in the seismicity rates at different magnitude cutoffs as well as simple statistics indicate that changes in the reporting and/or detecting of events have taken place at different times. We have found that the most significant changes at the $M>4.0$ level took place around the years 1940, 1968 and 1987. Other less significant changes occurred during 1960, 1983 and 1992. Comparing the rates obtained for the intervals bounded by the aforementioned dates we were able to determine that a correction to the magnitudes in the period 1968 to 1987 for the overall data set may be warranted in order to match the frequency-magnitude distribution which is obtained using current data in the interval 1987 - 2004. When separating shallow from intermediate and deep events, however, it was clear that most of the changes found before affected mainly the deep events $(z>40 \mathrm{~km})$, and that the rate of shallow seismicity has been surprisingly homogeneous for $M>4.4$ since 1940 notwithstanding the station coverage of that period.

Making a comparison of rates for shallow seismicity for the intervals bounded by the aforementioned dates, it was found that the FMD's match each other well from a particular magnitude onwards, indicating that the only change introduced by instrumentation has been in the minimum magnitude of completeness which has been steadily lowered. A systematic evaluation of minimum magnitude of completeness for shallow seismicity yielded values of $M_{c}=$ 4.4 for the interval 1940 to $1968, M_{c}=3.9$ for the interval 1968 to 1987 and $M_{c}=2.6$ for the current stage from 1987 to 2004.

Comparing the rates for the intervals analyzed before for the intermediate and deep events, we found similar behavior as for the whole catalogue again supporting the notion that the most conspicuous changes affected mostly these deeper data. Applying the $b$-value fitting technique, we found a magnitude transformation which allows for the FMD of the 1940 to 1968 interval to match that for the 1968 to 1987 . The comparison between rates in the interval 1968 to 1987 with that of 1987 to 2004 indicated that a simple shift of 0.2 units applied to magnitudes of data in the former interval may be useful in matching current statistics. The evaluation of $M_{c}$ for the intervals discussed yielded values of 5.5 for data in the interval 1940 to $1968,4.0$ for 1968 to 1987 and 3.6 was found a suitable estimate for current practice starting in 1987.

The variation of $M_{\mathrm{c}}$ with time was investigated resulting in different averages corresponding to the intervals discussed above, which in general show an improvement over time. $M_{\mathrm{c}}$ calculated from the maximum curvature of the non- 
cumulative frequency distribution for the period 1940 to 1968 fluctuated between 3.5 and 4.3 for the shallow seismicity as opposed to $4.2 \pm 0.1$ which was obtained for intermediate and deep events. For the interval 1968 to 1987 we found that for the shallow events $3.5 \leq M_{\mathrm{c}} \leq 4.0$ up to around 1978 but after this date it was considerably improved to values of less than 2.0 , although later fluctuations from 1984 to about 1986 caused it to oscillate between that value and pre-1978 estimates. The deeper seismicity for the same interval showed $M_{\mathrm{c}}=3.9 \pm 0.1$ with some fluctuations from 1978 to 1982 when values were again largely reduced to close to 2.0. For the last interval, from 1987 till today, we found $1.9 \leq M_{\mathrm{c}} \leq 2.7$ for the shallow seismicity while the mean of the deeper events was obtained as $3.0 \pm 0.3$. Using a least squares fit to the frequency-magnitude distribution for the latest period we get $M_{\mathrm{c}}=3.7$ for shallow events and 3.6 for the intermediate and deep range of the catalogue. The difference between estimates may be due to a bimodal linear trend in the distributions.

A likely explanation for the large changes in the magnitude of completeness for $z<40 \mathrm{~km}$ events from 1975 is the installation of dense, highly sensitive microearthquake networks in parts of the country around that time. The Wellington microearthquake network was installed in June 1975, and continues operation today. The Pukaki microearthquake network in the central South Island was also installed in June 1975, and it operated until November 1983. Equipment from the Pukaki network was then moved to Hawke's Bay in the North Island, where it recommenced operation in March 1987. A microearthquake network also operated in the Clyde region of the South Island from December 1986 until May 1996. As small earthquakes were located with these highly sensitive networks and these were added to the seismicity catalogue, the drop in magnitude of completeness from 1975 is to have been expected. Similarly, the rise in magnitude of completeness from 1984-1986 most likely reflects the loss of a microearthquake network in the South Island during this period.

The change in the magnitude-frequency distribution of the subcrustal events in 1987 is also likely to be due to a change in station distribution at that time. Most of the subcrustal events in the catalogue occur in the so-called "main seismic region" associated with subduction of the Pacific plate at the Hikurangi margin. Because of the known heterogeneity of upper mantle structure in this region, amplitudes from stations west of the volcanic region and south of the main seismic region are not used in the determination of magnitudes (Haines, 1981). The installation of the Hawke's Bay microearthquake network in 1987 effectively increased the density of stations in the shallow part of the subduction zone. As it is well known that earthquake waves suffer relatively little attenuation travelling up the subducted plate to this region, an upward bias in magnitudes of subcrustal earthquakes from this time is not unexpected.

\section{ACKNOWLEDGEMENTS}

We are grateful with colleagues at GNS since many fruitful discussions provided strong basis for this paper. In particular we would like to thank Matt Gerstenberger, Mark Stirling, Russell Robinson, Lorena Cowan and Rafael Benites. UNAM-DGAPA provided funding which made possible the exchange between both institutions. This article was written while one of the authors (R.Z.) was a visiting scientist for a year as sabbatical leave at the Institute of Geological and Nuclear Sciences Ltd. so help and support of GNS managers, staff and colleagues during his stay is gratefully acknowledged. The comments of P. McGinty led to an improved version of this manuscript.

\section{REFERENCES}

Anderson, H.J. and. Webb, T.H. (1994). "New Zealand seismicity; patterns revealed by the upgraded National Seismic Network", N.Z J. Geol. Gephys., 37, 477-493.

Dowrick, D., and Rhoades, D. (1998). "Magnitudes of New Zealand earthquakes, 1901-1993". Bull. New Zealand Nat. Soc. Earthq. Eng, 31, 260-280.

Gomberg, J. (1991). "Seismicity and detection/location threshold in the southern Great Basin seismic network", $J$. Geophys. Res., 96 (B10), 16,401-16,414.

Habermann, R.E. (1982). "Consistency of teleseismic reporting since 1963”. Bull. Seism. Soc. Am., 72, 93-112.

Habermann, R.E. (1983). "Teleseismic detection in the Aleutian Island Arc", J. Geophys. Res., 88, 5056-5064.

Habermann, R.E. and Wyss M. (1984). "Background seismicity rates and precursory seismic quiescence: Imperial Valley, California", Bull. Seism. Soc. Am., 74, 1743-1755.

Haines, A.J. (1981). "A local magnitude scale for New Zealand earthquakes”, Bull. Seism. Soc. Am., 71, 275-294.

McGinty, P, (2001). "Preparation of the New Zealand earthquake catalogue for probabilistic seismic hazard analysis", Bull. New Zealand Nat. Soc. Earth. Eng., 34, 6067.

Reasenberg, P. A., (1985). "Second-order moment of central California seismicity”, 1969-1982, J. Geophys. Res, 90, 5479-5495.

Smith, W., (2000). "Precursory changes in mean magnitudes: a systematic examination of the New Zealand catalogue", Institute of Geological and Nuclear Sciences Ltd., File Report 2000/2, 36pp.

Wiemer, S., (2001). "A software package to analyze seismicity: ZMAP”. Seism. Res. Let., 72, 3, 373-382. 
Wiemer, S. and Wyss M. (2000), "Minimum magnitude of completeness in earthquake catalogs: examples from Alaska, the western United States and Japan". Bull. Seism. Soc.,

Amer., 90, 859-869.

Wiemer, S., and Zúñiga F.R. (1994), "ZMAP - a software package to analyze seismicity", EOS, Transactions, Am. Geophys. U., 75, 456.

Wyss, M. (1991), "Reporting history of the central Aleutians seismograph network and the quiescence preceding the 1986 Andreanof Island earthquake”. Bull. Seism. Soc., Amer., 81, 1231-1254.

Wyss, M. and Burford R.O. (1985). "Current episodes of seismic quiescence along the San Andreas fault between San Juan Bautista and Stone Canyon, California: Possible Precursors to local moderate mainshocks?", U.S. Geol. Survey, open file report 85-745, 367-426.

Zúñiga, F.R (1989). "A study of the homogeneity of the NOAA earthquake data file in the Mid-America region by the magnitude signature technique". Geofisica Internacional, 28, 103-119.

Zúñiga, F.R. and Wyss M. (1995). "Inadvertent changes in magnitude reported in earthquake catalogs: Influence on bvalue estimates", Bulletin of the Seismological Society of America, 85, 1858-1866.

Zúñiga, F.R, Valdés C.M. and Reyes M. (2000). "A general overview of the catalog of recent seismicity compiled by the Mexican Seismological Survey". Geofísica Internacional, 39, 161-170. 